\title{
Stability of body mass index in Australian children: a prospective cohort study across the middle childhood years
}

\author{
Kylie Hesketh ${ }^{1, *}$, Melissa Wake ${ }^{1}$, Elizabeth Waters ${ }^{1}$, John Carlin ${ }^{2}$ and David Crawford ${ }^{3}$ \\ ${ }^{1}$ Centre for Community Child Health, Murdoch Childrens Research Institute \& University of Melbourne, \\ Royal Children's Hospital, Flemington Road, Parkville, Victoria 3052, Australia: ${ }^{2}$ Clinical Epidemiology and \\ Biostatistics Unit, Murdoch Childrens Research Institute \& University of Melbourne, Royal Children's Hospital, \\ Melbourne, Australia: ${ }^{3}$ Centre for Physical Activity and Nutrition Research, School of Health Sciences, \\ Deakin University, Melbourne, Australia
}

Submitted 10 July 2003: Accepted 3 September 2003

\begin{abstract}
Objective: To investigate the prevalence and incidence of overweight and obesity, the frequency of overweight resolution and the influence of parental adiposity during middle childhood.

Design: As part of a prospective cohort study, height and weight were measured in 1997 and 2000/2001. Children were classified as non-overweight, overweight or obese based on standard international definitions. Body mass index (BMI) was transformed into age- and gender-specific $Z$-scores employing the LMS method and 2000 growth chart data of the Centers for Disease Control and Prevention. Parents self-reported height and weight, and were classified as underweight, healthy weight, overweight or obese based on World Health Organization definitions.

Setting: Primary schools in Victoria, Australia.

Subjects: In total, 1438 children aged 5-10 years at baseline.

Results: The prevalence of overweight and obesity increased between baseline (15.0 and 4.3\%, respectively) and follow-up (19.7 and 4.8\%, respectively; $P<0.001$ for increase in overweight and obesity combined). There were 140 incident cases of overweight (9.7\% of the cohort) and 24 of obesity ( $1.7 \%$ of the cohort); only $3.8 \%$ of the cohort (19.8\% of overweight/obese children) resolved to a healthy weight. The stability of child adiposity as measured by BMI category ( $84.8 \%$ remained in the same category) and BMI $Z$-score $(r=0.84$; mean change $=-0.05)$ was extremely high. Mean change in BMI $Z$-score decreased with age (linear trend $\beta=0.03,95 \%$ confidence interval $0.01-0.05)$. The influence of parental adiposity largely disappeared when children's baseline BMI was adjusted for.

Conclusions: During middle childhood, the incidence of overweight/obesity exceeds the proportion of children resolving to non-overweight. However, for most children adiposity remains stable, and stability appears to increase with age. Prevention strategies targeting children in early childhood are required.
\end{abstract}

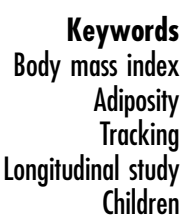

Overweight/obesity is a chronic health condition afflicting a large, and growing, number of children ${ }^{1}$. However, many parents express no concern about their overweight or obese child's weight ${ }^{2,3}$ and do not perceive it to be a health problem ${ }^{3}$. This may be due to the perception that their children are happy and healthy or will eventually 'grow out of t' $^{4}$. Yet we know that overweight and obese children are more likely to suffer from psycho-emotional concerns $^{1}$ and obese children, specifically, are at greater risk for numerous physical health problems ${ }^{1}$.

A growing body of evidence indicates that many overweight and obese children do not 'grow out of it'. Findings from longitudinal studies consistently show that adiposity in childhood is correlated with adiposity in adolescence and adulthood ${ }^{5}$. Obese children have a high likelihood of their obesity persisting into adulthood ${ }^{6}$, particularly if one or both parents is/are overweight or obese $^{7}$. US data suggest that a child who is overweight or obese during middle childhood has 10 times greater odds of being an obese adult than a child who is not overweight $^{8}$. More than $50 \%$ of overweight or obese children are still classified as overweight or obese in later childhood or adolescence ${ }^{9-12}$.

Evidence on the proportion of children whose overweight or obesity resolves is considerably more limited, and studies provide conflicting results ${ }^{13-15}$. Various 
definitions of overweight resolution, small sample sizes ${ }^{13}$, poor retention rates ${ }^{13,14}$ and different populations make the comparison of results across studies difficult. Yet these children are of particular interest, as they may offer insights into prevention and management strategies.

Although the prevalence of childhood overweight and obesity has been widely reported ${ }^{15}$, information on the incidence is lacking. For children in the middle childhood years the incidence of overweight and obesity appears to be relatively low ${ }^{13,14}$. However, the accuracy of these estimates is uncertain as the studies upon which these data are based had very low retention rates.

In this prospective cohort study, we determined the prevalence and incidence of overweight and obesity for a cohort of Australian children aged 5-10 years at recruitment and followed them up, 3 years later. We investigated the stability of BMI over the 3 years, and the proportions of children maintaining overweight/obesity, developing overweight or obesity and resolving from overweight/obesity to a healthy weight. We also considered the influence of parental body mass index (BMI) on the stability of children's BMI over time. As far as we are aware, this is one of only a few population-based studies to examine these issues in children, and the first to explore these issues in Australian children.

\section{Methods}

\section{Sample}

The baseline sample was drawn from the Health of Young Victorians Study (HOYVS), conducted between September and December 1997. HOYVS employed a two-stage stratified random sampling design to draw a sample of children representative of the state of Victoria, Australia. In the first stage of sampling, 24 primary schools were selected, with a probability proportional to size of school enrolment, to be representative of the different school sectors (government, Catholic, independent) and the geographic distribution of the state. In the second stage, one class at each year level (preparatory to grade six) at each school was randomly selected for the study. Children in grades preparatory to three (aged 5-10 years) who provided data in the initial survey were included in the follow-up study, conducted between October 2000 and June 2001. The average time between baseline and followup assessments was 3.2 (standard deviation (SD) 0.2) years. Both studies were approved by the Royal Children's Hospital Ethics in Human Research Committee, the Victorian Department of Education and the Catholic Education Office.

\section{Measures}

At both baseline and follow-up children had their height and weight measured by trained fieldworkers. Investigation of anthropometric reliability found no evidence of systematic bias for intra-rater or inter-rater comparisons.
BMI was calculated as weight (in $\mathrm{kg}$ ) divided by the square of height (in $\mathrm{m}$ ). Children were categorised into three BMI categories (non-overweight, overweight and obese) according to the gender- and age-specific cut-off points developed by the International Obesity Task Force ${ }^{16}$. Further, BMI was transformed into externally standardised $(Z)$ scores based on gender and exact age, to adjust for right skew in the BMI distribution and physiological changes that occur in child BMI with age. We employed the LMS method ${ }^{17}$ using the 2000 growth chart data ${ }^{18}$ from the Centers for Disease Control and Prevention to perform the BMI transformation.

Parents self-reported the height and weight of the child's biological mother and father, from which BMI was calculated. Parents were categorised into four BMI categories based on World Health Organization definitions ${ }^{1}$ : underweight $\left(<18.5 \mathrm{~kg} \mathrm{~m}^{-2}\right)$, healthy weight $\left(18.5-24.9 \mathrm{~kg} \mathrm{~m}^{-2}\right)$, overweight $\left(25-29.9 \mathrm{~kg} \mathrm{~m}^{-2}\right)$ and obese $\left(\geq 30 \mathrm{~kg} \mathrm{~m}^{-2}\right)$.

\section{Analyses}

Characteristics of children retained and those lost to follow-up were compared using the independent samples $t$-test and Pearson's chi-square statistic. Change in the prevalence of overweight and obesity was examined using McNemar's chi-square statistic. Gender and age differences in proportions were assessed using Pearson's chisquare statistic. Due to small numbers, children aged 10 years at baseline were combined with those aged 9 years for all analyses by age group. Logistic regression analyses were used to estimate the relative odds of being classified as overweight or obese at follow-up, based on a child's BMI category at baseline.

We further examined the stability of BMI over time using continuous BMI $Z$-score data. Pearson correlation coefficients were used to assess the relationship between BMI $Z$-scores at baseline and follow-up. Change in BMI $Z$-score for each subject was calculated as BMI $Z$-score at followup minus BMI $Z$-score at baseline. A positive change in BMI $Z$-score indicates that a child's BMI relative to their age is higher at follow-up than at baseline. Change in BMI $Z$-score exceeded two standard deviations for five children. Exclusion of these children as possible outliers did not alter the results, and hence they were retained.

Possible differences in change in BMI $Z$-score by gender or age group were explored using independent samples $t$-test and linear regression for trend analyses, respectively. Mean BMI $Z$-score for each child was calculated by averaging its baseline and follow-up BMI $Z$-score values. Unlike baseline BMI $Z$-score, the association between mean BMI $Z$-score and change in BMI $Z$-score is not influenced by the strength of correlation between baseline and follow-up BMI $Z$-score, thus overcoming the issue of regression to the mean ${ }^{19}$.

To investigate relationships between child and parental adiposity, logistic regression analyses (unadjusted and 
adjusted for baseline child BMI $Z$-score) were conducted to consider the odds of a child being overweight/obese at follow-up, based on their parents' BMI categories at baseline. Further logistic regression analyses considered the odds of a non-overweight child developing overweight/obesity based on their parents' BMI categories at baseline, and the odds of an overweight/obese child resolving to non-overweight. Finally, regression analyses were used to determine whether a linear trend existed between change in children's BMI $Z$-score and parental BMI category at baseline.

\section{Results}

\section{Sample}

The response rate for the baseline study was $83.2 \%$ (1943/2336) of children in grades preparatory to three (ages 5 to 10 years). Of the children who provided data in the baseline study, 374 did not participate in the follow-up study: 28 could not be identified, 43 had moved interstate, 11 had moved overseas, 122 had transferred schools and their whereabouts was unknown, and a further 170 declined to participate. Thus, 1569 children provided data in the follow-up study, a retention rate of $80.8 \%$ of the baseline cohort. A further 131 children were excluded from analyses for this paper because of incomplete data.

The remaining 1438 children did not differ from the 505 lost to follow-up or excluded in terms of mean age or gender. However, children retained in the cohort had a lower mean BMI at baseline (16.9 vs. $17.5 \mathrm{~kg} \mathrm{~m}^{-2}$; $P<0.001)$ than children lost to follow-up, and a smaller proportion were classified as overweight or obese (15.0 vs. $18.5 \%$ overweight and 4.3 vs. $10.3 \%$ obese; $P<0.001$ ).

Prevalence and incidence of overweight and obesity The prevalence of overweight and obesity was 15.0 and $4.3 \%$, respectively, at baseline, increasing to 19.7 and $4.8 \%$ at follow-up (Table 1). The increase in the total proportion of children classified as overweight/obese was significant $(P<0.001)$. A slightly higher proportion of females than males were classified as overweight (16.7 vs. $13.4 \%)$ or obese (4.9 vs. 3.8\%) at baseline and at follow-up (20.7\% overweight and $5.4 \%$ obese females vs. $18.7 \%$ overweight and $4.2 \%$ obese males). However, the gender difference was not significant at either time (baseline: $P=0.11$; follow-up: $P=0.30)$.

Most children (84.8\%) remained in the same BMI category at baseline and follow-up. Only $11.1 \%$ of children who were non-overweight at baseline changed BMI category. However, $33.8 \%$ of overweight and $27.4 \%$ of obese children changed category between baseline and follow-up. Of the 219 children who changed BMI category, a larger proportion moved into a higher BMI category (68.0\%) than moved into a lower BMI category (32.0\%). In total, there were 140 incident cases of overweight ( $9.7 \%$ of the total sample) and 24 incident cases of obesity (1.7\% of the total sample). Males and females contributed almost identical numbers of incident cases.

Fifty-five children (3.8\% of the total sample; $19.8 \%$ of those classified as overweight or obese at baseline) resolved their weight problem, to be classified as nonoverweight at follow-up. A slightly higher proportion of females (20.6 vs. 18.7\% of males) and of younger children (21.9\% of 5 -year-olds, $27.0 \%$ of 6 -year-olds, $17.6 \%$ of 7 -year-olds, $16.2 \%$ of 8 -year-olds and $17.1 \%$ of $9-10$-yearolds) resolved their weight problem. However, neither the gender nor the age difference was statistically significant ( $P=0.69$ and 0.54 , respectively).

Logistic regression analyses showed that a child who was classified as overweight at baseline had 25 times greater odds (95\% confidence interval (CI) 17-35) of being classified as either overweight or obese at follow-up than a child who was non-overweight at baseline. A child who was classified as obese at baseline had 240 times greater odds (95\% CI 58-993) of being classified as overweight/obese at follow-up than a child who was nonoverweight at baseline. There was no difference in the magnitude of increased odds by gender or age group.

\section{Stability of BMI Z-scores over time}

There was a high correlation $(r=0.84)$ between BMI $Z$-score at baseline and follow-up for the sample overall (Fig. 1), for males $(r=0.83)$ and for females $(r=0.85)$, and for all age groups (all $r>0.80$ ). The average change in BMI $Z$-score between baseline and follow up was -0.05 (SD 0.52), suggesting that the total increases in BMI $Z$-score were equivalent to the total decreases across the

Table 1 Numbers and proportions of children in each body mass index (BMI) category at baseline and follow-up, and mean change in BMI Z-score for each group

\begin{tabular}{|c|c|c|c|c|}
\hline \multirow[b]{2}{*}{ BMI category at baseline } & \multicolumn{4}{|c|}{ BMI category at follow-up } \\
\hline & Non-overweight & Overweight & Obese & Total \\
\hline Non-overweight & $1031(88.9)[-0.11]^{\star}$ & 125 (10.8) [0.60] & $4(0.3)[1.19]$ & $1160(100.0)[-0.03]$ \\
\hline Overweight & $53(24.5)[-0.65]$ & $143(66.2)[0.00]$ & $20(9.3)[0.35]$ & $216(100.0)[-0.12]$ \\
\hline Obese & $2(3.2)[-1.82]$ & $15(24.2)[-0.50]$ & $45(72.6)[-0.06]$ & $62(100.0)[-0.23]$ \\
\hline Total & $1086(75.5)[-0.14]$ & $283(19.7)[0.24]$ & $69(4.8)[0.15]$ & $1438(100.0)[-0.05]$ \\
\hline
\end{tabular}

${ }^{*}$ Values are expressed as $n(\%)$ [mean change in BMI Z-score]. Bold denotes children who did not change category. 


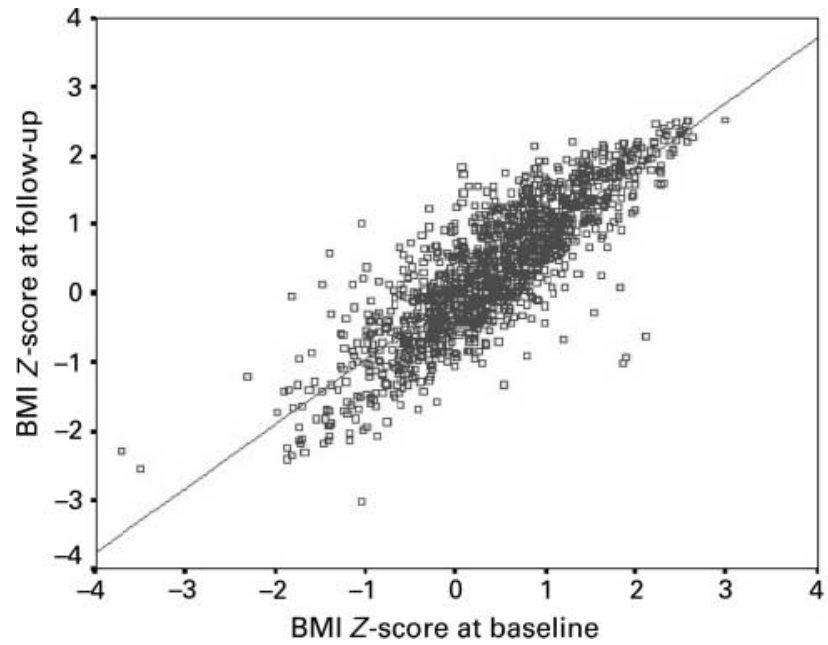

Fig. 1 Scatter plot of body mass index (BMI) Z-scores at baseline and follow-up

sample. Although $70.0 \%$ of BMI $Z$-scores changed by no more than half a standard deviation $(0.5 Z$-score) between baseline and follow-up, there was a wide range $(-2.85$ to $2.05)$. Change in BMI $Z$-score was similar for males and females $(P=0.61)$. However, mean change in BMI $Z$-score was greater in the younger age groups $(-0.14$, $-0.08,-0.04,-0.03$ and -0.01 for $5-, 6-, 7-, 8$ - and $9-10-$ year-olds, respectively; linear trend $\beta=0.03,95 \% \mathrm{CI}$ $0.01-0.05)$, even after adjusting for mean BMI $Z$-score $(\beta=0.03,95 \%$ CI $0.01-0.05)$.

Although there was no mean change in BMI $Z$-score for the sample overall, there was a weak positive correlation between change in BMI $Z$-score and mean BMI $Z$-score (the average of the baseline and follow-up BMI $Z$-scores) $(r=0.18)$. This indicates a tendency for greater increases among heavier children. However, this can be explained by the fact that the variation in BMI $Z$-scores increased between baseline and follow-up (SD 0.86 and 0.95, respectively) ${ }^{19}$.

\section{Association with parental BMI}

Cross-sectionally, children had a greater risk of being overweight/obese at baseline if either parent was overweight (odds ratio $(\mathrm{OR})=2.23,95 \%$ CI $1.60-3.12$ for maternal overweight; OR $=1.39,95 \%$ CI 1.01-1.91 for paternal overweight) or obese (OR $=3.23,95 \%$ CI 2.18 4.80 for maternal obesity; OR $=2.49,95 \%$ CI $1.62-3.84$ for paternal obesity). Similarly, children had a greater risk of being overweight/obese at follow-up if either parent was overweight or obese at baseline (Table 2). This relationship persisted for maternal obesity only once baseline child BMI $Z$-score was accounted for.

Children who were overweight/obese at baseline had lesser odds of resolving to non-overweight at follow-up if they had an obese mother ( $\mathrm{OR}=0.36,95 \% \mathrm{CI} 0.13-0.98)$. This relationship was weaker once child BMI $Z$-score at
Table 2 Odds ratio (95\% confidence interval $(\mathrm{Cl})$ ) for children being overweight/obese at follow-up, based on parental body mass index (BMI) category at baseline

\begin{tabular}{|c|c|c|c|}
\hline & $n$ & $\begin{array}{l}\text { Odds ratio } \\
(95 \% \mathrm{Cl})- \\
\text { unadjusted }\end{array}$ & $\begin{array}{c}\text { Odds ratio } \\
(95 \% \mathrm{Cl})-\text { adjusted } \\
\text { for baseline } \\
\text { child BMI Z-score }\end{array}$ \\
\hline \multicolumn{4}{|c|}{ Maternal BMI category at baseline } \\
\hline Underweight & 52 & $0.3(0.1-0.9)$ & $0.3(0.1-1.2)$ \\
\hline Healthy weight & 824 & 1.0 & 1.0 \\
\hline Overweight & 254 & $2.0(1.5-2.9)$ & $1.3(0.8-2.0)$ \\
\hline Obese & 139 & $3.6(2.5-5.2)$ & $1.8(1.1-3.1)$ \\
\hline \multicolumn{4}{|c|}{ Paternal BMI category at baseline } \\
\hline Underweight & 8 & $2.6(0.6-10.9)$ & $2.0(0.2-17.8)$ \\
\hline Healthy weight & 491 & 1.0 & 1.0 \\
\hline Overweight & 551 & $1.6(1.2-2.1)$ & $1.4(0.9-2.0)$ \\
\hline Obese & 139 & $2.3(1.5-3.5)$ & $1.0(0.6-1.9)$ \\
\hline
\end{tabular}

baseline was accounted for (OR $=0.46,95 \%$ CI $0.16-$ 1.33). Overweight/obese children with an overweight father had lesser odds of resolving to non-overweight at follow-up than did children whose father was a healthy weight $(\mathrm{OR}=0.47,95 \%$ CI 0.23-0.98), even after controlling for child BMI $Z$-score at baseline $(\mathrm{OR}=0.44$, 95\% CI 0.20-0.98).

The relationship between parental BMI category at baseline and change in children's BMI $Z$-score was also considered (Table 3). There was a linear trend in change in BMI Z-score for girls across baseline maternal BMI categories $(\beta=0.10,95 \%$ CI $0.05-0.15)$; girls whose mothers were obese at baseline had the greatest upward change in BMI $Z$-score over the 3 years. This was less evident for boys $(\beta=0.05,95 \% \mathrm{CI}-0.01,0.10)$. No linear trend was observed across fathers' BMI categories. For those children with baseline BMI data for both parents ( $n=1155$; 80\%), there was a linear association between number of parents overweight/obese at baseline and change in children's BMI $Z$-score for females $(\beta=0.08$, 95\% CI 0.03-0.14) but not for males ( $\beta=0.02,95 \%$ CI $-0.04,0.08)$. A stronger association was observed for number of parents obese at baseline and change in children's BMI $Z$-score, which was again clearly significant for females $(\beta=0.13,95 \%$ CI $0.04-0.21)$ but weaker for males $(\beta=0.08,95 \% \mathrm{CI}-0.01,0.17)$.

\section{Discussion}

This study of a representative sample of Australian children indicates that BMI remains relatively stable during middle childhood. The vast majority of children in this study remained in the same BMI category between baseline and follow-up, and there was no mean change in BMI $Z$-score for the sample overall. However, despite the majority of children having stable relative BMI over the 3year period of this study, there were a small number of children whose relative BMI did change over time. When classified using internationally defined criteria, the 
Table 3 Relationship between parental body mass index (BMI) at baseline and change in child BMI Z-score

\begin{tabular}{|c|c|c|c|}
\hline & \multirow[b]{2}{*}{$n$} & \multicolumn{2}{|c|}{ Mean change in BMI Z-score } \\
\hline & & Males & Females \\
\hline \multicolumn{4}{|c|}{ Maternal BMI category at baseline } \\
\hline Underweight & 52 & -0.09 & -0.25 \\
\hline Healthy weight & 824 & -0.06 & -0.11 \\
\hline Overweight & 254 & -0.10 & -0.02 \\
\hline Obese & 139 & 0.09 & 0.08 \\
\hline Linear trend $(95 \% \mathrm{Cl})$ & & $0.05(-0.01-0.10)$ & $0.10^{\star * *}(0.05-0.15)$ \\
\hline \multicolumn{4}{|c|}{ Paternal BMI category at baseline } \\
\hline Underweight & 8 & -0.21 & -0.01 \\
\hline Healthy weight & 491 & -0.07 & -0.10 \\
\hline Overweight & 551 & -0.05 & -0.06 \\
\hline Obese & 139 & -0.02 & 0.00 \\
\hline Linear trend $(95 \% \mathrm{Cl})$ & & $0.03(-0.04-0.09)$ & $0.04(-0.02-0.10)$ \\
\hline \multicolumn{4}{|c|}{ Number of overweight/obese parents at baseline } \\
\hline Neither & 374 & -0.03 & -0.13 \\
\hline One & 537 & -0.11 & -0.08 \\
\hline Both & 244 & 0.03 & 0.04 \\
\hline Linear trend $(95 \% \mathrm{Cl})$ & & $0.02(-0.04-0.08)$ & $0.08^{\star *}(0.03-0.14)$ \\
\hline \multicolumn{4}{|c|}{ Number of obese parents at baseline } \\
\hline Neither & 924 & -0.08 & -0.10 \\
\hline One & 199 & 0.00 & 0.05 \\
\hline Both & 32 & 0.10 & 0.05 \\
\hline Linear trend $(95 \% \mathrm{Cl})$ & & $0.08(-0.01-0.17)$ & $0.13^{\star \star}(0.04-0.21)$ \\
\hline
\end{tabular}

majority of changes between BMI category were towards a heavier category. Together, these findings suggest that we cannot be complacent about childhood weight issues.

The increased prevalence of overweight and obesity observed at follow-up may be a reflection of the secular changes occurring within our community ${ }^{20}$ or may be a result of the increasing ages of the children studied ${ }^{21}$. However, the observed changes across BMI categories could equally be an artefact of applying cut-off points derived from cross-sectional datasets to these longitudinal data. Unlike adult cut-off points, the BMI values used to classify overweight and obesity in children vary by age and gender to account for the variations in growth that occur for children at different ages. However, if the growth curve of the population from which the cut-off points were derived does not match the growth curve of this cohort, reported changes in BMI category over the course of the study may reflect differences in patterns of growth, rather than true changes in adiposity. Similarly, the slight increase observed in variance of the BMI $Z$-score might be an artefact of age-related variation in the extent to which growth in our population resembles the US population from which the reference standard for converting BMI to BMI $Z$-score was derived.

While only a small proportion of the cohort showed resolution of overweight or obesity, these children represented almost one-fifth of the children classified as overweight or obese at baseline. This is encouraging and indicates that some children are able to slow their weight gain relative to height growth to become non-overweight. We do not know how many of these children sought treatment for their weight problems, but the lack of formal treatment options, and the limited effectiveness of those available $^{22}$, make it likely that most of these weight problems resolved without formal intervention. Such children may provide clues to characteristics or behaviours facilitating relative weight reduction, which in turn may present promising targets for prevention and intervention strategies aimed at reducing the incidence and prevalence of childhood overweight and obesity.

Parental BMI was associated with children's risk of being overweight or obese at baseline and follow-up, and with change in BMI $Z$-score. It may have influenced whether an overweight child maintained overweight or resolved to a healthier BMI. Relationships were stronger for maternal than paternal BMI, and for female than male children. However, all associations between child and parental BMI largely disappeared when children's baseline BMI was accounted for. This observation is in line with findings from previous studies ${ }^{8}$ and suggests that while parents' adiposity may be important in setting children on a weight trajectory, by the middle childhood years the influence of parental adiposity is secondary to the child's own weight history. As such, whilst it may be useful to take parental adiposity into consideration when targeting children to be involved in intervention programmes for overweight, the child's own weight history may be of greater importance. 
The mean BMI of children lost to follow-up in this study was higher than the mean BMI of those retained in the cohort. Whilst this reduced the proportion of overweight/ obese children available for study, implications for the overall findings and conclusions of the study are likely to be negligible. A tendency for heavier children in this sample to show greater increases in relative BMI over time, although largely explained by the increased variation in BMI Z-scores between baseline and follow-up, may warrant further investigation. However, as the majority of children, regardless of baseline weight status, showed little change in relative BMI over time, there is no reason to suspect that those lost to follow-up would show greater change.

The findings of this study suggest that effective interventions are needed for overweight children in early childhood. Without intervention, relatively few resolve to a healthy weight in the middle childhood years, leaving the great majority to carry their weight problems into adolescence. While being overweight in the middle childhood years increases a child's risk of being overweight in adulthood, the risk is even higher for those who are overweight as adolescents ${ }^{8}$. Tackling the problem in those children who are already overweight, whilst important, is unlikely to stem the rising prevalence of overweight and obesity. Population prevention strategies aimed at preventing children from initially becoming overweight or obese are of paramount importance.

Our findings indicate that 5- and 6-year-old children experience greater reduction in adiposity than their older peers. This suggests that young children may be more malleable, with the precipitants of weight stability not yet firmly entrenched. School entry provides a unique opportunity for population intervention strategies. The school environment provides the first forum since the early infant period through which almost all children (and their parents) are easily accessible. However, with almost one-fifth of the children in this study already overweight at school entry, research to ascertain the timing of onset and possible precipitating factors, and prevention approaches targeting children prior to school entry, are required.

\section{Acknowledgements}

This study was supported by grants from the National Heart Foundation, Financial Markets for Children, and Murdoch Children's Research Institute. K.H. is supported by a National Health and Medical Research Council Public Health Postgraduate Scholarship. D.C. is supported by a National Health and Medical Research Council/National Heart Foundation Career Development Award. We would like to acknowledge the contribution to data collection of Bibi Gerner, Louisa Salmon, Naomi Paine, Susan Gallagher, Jennifer van Gemert, Sarah Barker and Winnie Lau.

\section{References}

1 World Health Organization (WHO). Obesity: Preventing and Managing the Global Epidemic. Geneva: WHO, 1998.

2 Wake M, Salmon L, Waters E, Wright M, Hesketh K. Parentreported health status of overweight and obese Australian primary school children: a cross-sectional population survey. International Journal of Obesity and Related Metabolic Disorders 2002; 26: 717-24.

3 Young-Hyman D, Herman LJ, Scott DL, Schlundt DG. Care giver perception of children's obesity-related health risk: a study of African American families. Obesity Research 2000; 8 : 241-8.

4 Jain A, Sherman SN, Chamberlin DL, Carter Y, Powers SW, Whitaker RC. Why don't low-income mothers worry about their preschoolers being overweight? Pediatrics 2001; 107: $1138-46$.

5 Parsons TJ, Power C, Logan S, Summerbell CD. Childhood predictors of adult obesity: a systematic review. International Journal of Obesity and Related Metabolic Disorders 1999; 23(Suppl. 8): S1-S107.

6 Serdula MK, Ivery D, Coates RJ, Freedman DS, Williamson DF, Byers T. Do obese children become obese adults? A review of the literature. Preventive Medicine 1993; 22 167-77.

7 Lake JK, Power C, Cole TJ. Child to adult body mass index in the 1958 British birth cohort: associations with parental obesity. Archives of Disease in Childhood 1997; 77: 376-81.

8 Whitaker RC, Wright JA, Pepe MS, Seidel KD, Dietz WH. Predicting obesity in young adulthood from childhood and parental obesity. New England Journal of Medicine 1997; 337: 869-73.

9 Sugimori H, Yoshida K, Miyakawa M, Izuno T, Takahashi E, Nanri S. Temporal course of the development of obesity in Japanese school children: a cohort study based on the Keio Study. Journal of Pediatrics 1999; 134: 749-54.

10 Kelly JL, Stanton WR, McGee R, Silva PA. Tracking relative weight in subjects studied longitudinally from ages 3 to 13 years. Journal of Paediatrics and Child Health 1992; 28: 158-61.

11 Angelico F, Del Ben M, Barbato A, Pannozzo F, Volpe R, Urbinati $G$, et al. Eleven-year tracking of established cardiovascular risk factors in Italian school-aged children. Annali di Igiene 1997; 9: 193-200.

12 Katzmarzyk PT, Perusse L, Malina RM, Bouchard C. Sevenyear stability of indicators of obesity and adipose tissue distribution in the Canadian population. American Journal of Clinical Nutrition 1999; 69: 1123-9.

13 Maffeis C, Talamini G, Tato L. Influence of diet, physical activity and parents' obesity on children's adiposity: a fouryear longitudinal study. International Journal of Obesity and Related Metabolic Disorders 1998; 22: 758-64.

14 Mo-suwan L, Tongkumchum P, Puetpaiboon A. Determinants of overweight tracking from childhood to adolescence: a 5 y follow-up study of Hat Yai schoolchildren. International Journal of Obesity and Related Metabolic Disorders 2000; 24: 1642-7.

15 Booth ML, Wake M, Armstrong T, Chey T, Hesketh K, Mathur $\mathrm{S}$. The epidemiology of overweight and obesity among Australian children and adolescents, 1995-97. Australian and New Zealand Journal of Public Health 2001; 25 $162-9$

16 Cole TJ, Bellizzi MC, Flegal KM, Dietz WH. Establishing a standard definition for child overweight and obesity worldwide: international survey. British Medical Journal 2000; 320: 1240-3.

17 Cole TJ, Green PJ. Smoothing reference centile curves: the LMS method and penalized likelihood. Statistics in Medicine 1992; 11: 1305-19.

18 Kuczmarski RJ, Ogden CL, Grummer-Strawn LM, Flegal KM, 
Guo SS, Wei R, et al. CDC Growth Charts: United States. Advanced Data from Vital and Health Statistics No. 314. Bethesda, MD: National Center for Health Statistics, 2000.

19 Hayes RJ. Methods for assessing whether change depends on initial value. Statistics in Medicine 1998; 7: 915-27.

20 Booth M, Chey T, Wake M, Norton K, Hesketh K, Dollman J, et al. Change in prevalence of overweight and obesity among young Australians, 1969-1997. American Journal of Clinical Nutrition 2003; 77: 29-36.

21 Rudolf MC, Sahota P, Barth JH, Walker J. Increasing prevalence of obesity in primary school children: cohort study. British Medical Journal 2001; 322: 1094-5.

22 Summerbell CD, Ashton V, Campbell KJ, Edmunds L, Kelly S, Waters E. Interventions for treating obesity in children [Cochrane Review]. The Cochrane Library, Issue 3. Oxford: Update Software, 2003. 